\section{Co-existence of SLE and HIV: A Life Threatening Combina- tion}

\section{Mohammad Shariq Mukarram*, Muhammad Ishaq Ghauri}

Department of Internal Medicine, Jinnah Medical College Hospital, SR-6, Sector 7-A, Korangi Industrial Area, Karachi, Pakistan

\begin{abstract}
Background

The presence of SLE with HIV is a rare and a life threatening phenomenon. Very few cases have been reported throughout the world about this nearly fatal combination.

\section{Case presentation}

This is a case report of a 40 year old woman of Asian descent who presented to the rheumatology clinic with painful small joints of both hands and was diagnosed with SLE. She later presented with diarrhea along with weight loss and an oral thrush. On laboratory investigations she turned out to be HIV positive.

\section{Conclusion}

The simultaneous presence of SLE and HIV in a patient is rare, difficult to diagnose and treat. The diagnosis of SLE in our patient was according to the ACR criteria.

Keywords: Co-existence; Human immune deficiency virus; Systemic lupus erythematosus
\end{abstract}

\section{Introduction}

SLE is a complex, multi systemic, autoimmune disease that is characterized by the formation of antibodies directed against the components of the cell nucleus. The exact etiology of the disease is unknown [1]. SLE, an autoimmune disease, can occur despite loss of immune competence caused by HIV infection. According to the recent literature 55 cases of SLE in setting of HIV infection have been reported [2].

\section{Case Presentation}

A 40 year old married female, mother of one child, presented in the rheumatology clinic with the complaint of pain in small joints of both hands and photophobia for past 1 year. Pain was sudden in onset, symmetrical, gradually progressive and moderate to severe in intensity. There was presence of morning stiffness in both the hands that

${ }^{*}$ Corresponding author: Mohammad Shariq Mukarram, Department of Internal Medicine, Jinnah Medical College Hospital, SR-6, Sector 7-A, Korangi Industria Area, Karachi, Pakistan, Tel: +92 3343572852; Email: shariq.msm@gmail.com

Citation: Mukarram MS, Ghauri MI (2017) Co-existence of SLE and HIV: A Life Threatening Combination. J Clin Stud Med Case Rep 4: 038.

Received: December 8, 2016; Accepted: April 10, 2017; Published: April 25, 2017 lasted up to one and a half hour. She had been taking NSAIDS for her joint pain which proved to be of no benefit to her.

Patient also complained of massive hair loss during the course of her illness accompanied with oral ulcers, on and off. No history of any previous abortions or miscarriages was noted. There was no family history of any disease.

On examination of her joints, patient had pain and tenderness in her proximal interphalangeal and Meta carpo-phalangeal joints of both hands. Rest of the joints, when examined, seemed to be normal. Presence of a Malar rash, over the cheeks could clearly be appreciated.

Laboratory investigations showed Hemoglobin $12.5 \mathrm{~g} / \mathrm{dl}$, lymphocytes $0.9^{*} 10^{9} / \mathrm{L}$, platelets $170,000 / \mathrm{mcL}$ and an ESR of $80 \mathrm{~mm} / \mathrm{h}$. Rest of the baseline investigations were within normal limits. The patient underwent ANA profile which revealed high titers for ANA and positive Anti Smith $(8.96 \mathrm{U} / \mathrm{ml})$ antibodies. On further investigation Anti ds DNA was also positive. Complement levels could not be done due to unavailability.

Patient was diagnosed with SLE, according to the SLICC criteria and was discharged on Prednisolone and Hydroxychloroquine and was advised a CBC and an ESR.

She came for her follow up 2 months later with $\mathrm{CBC}$ showing the same number of lymphocytes $\left(0.9^{\star} 10^{9}\right)$, platelets $175,000 / \mathrm{mcL}$ and an ESR of $70 \mathrm{~mm} / \mathrm{h}$. Same treatment was continued.

Patient started to improve with her pain initially and was generally doing well till the next 4 months when she presented with one month of watery diarrhea and weight loss which resulted in hospital admission. Diarrhea was watery, copious in amount, foul smelling (6 to 7 episodes per day). It was associated with nausea, abdominal cramps, anorexia and low grade fever along with a weight loss of $1.5 \mathrm{lbs}$.

At the time of admission patient was pyretic, temperature $100^{\circ} \mathrm{F}$, pulse of 94 beats/min, respiratory rate of 18 breaths/min and blood pressure reading of 110/70mmhg. On physical examination she had a classic oral thrush indicating oropharyngeal candidiasis with some signs of dehydration. Abdominal examination revealed a non-distended abdomen with diffuse tenderness upon deep palpation. No viscera was however palpable. There was no evidence of shifting dullness, gut sounds were audible.

Respiratory, Cardiovascular and Central nervous system examinations were unremarkable. GCS was $15 / 15$ with all cranial nerves intact. Plantars were down going and the sensibility examination was normal with intact position, vibration and touch sensation.

Routine investigations showed hemoglobin of $12.5 \mathrm{~g} / \mathrm{dl}$, lymphocytes $0.8^{*} 10^{9}$ and an ESR of $90 \mathrm{~mm} / \mathrm{h}$. Serum creatinine $0.6 \mathrm{mg} / \mathrm{dl}$ and Urea $30 \mathrm{mg} / \mathrm{dl}$. Urine D/R showed 1-2 leukocytes and epithelial cells $(+++)$. Liver function tests showed total bilirubin of $0.52 \mathrm{mg} / \mathrm{dl}$ and SGPT 49 U/L. Serum Sodium was found to be $134 \mathrm{meq} / \mathrm{l}$, potassium $2.5 \mathrm{meq} / \mathrm{l}$, chloride $101 \mathrm{meq} / \mathrm{l}$ and bicarbonate $20 \mathrm{meq} / \mathrm{l}$. Stool examination showed presence of mucus with 8-10 leukocytes. A stool culture was sent which showed growth of Compylobacter Jejuni.

A clinical suspicion of HIV was made. Further workup revealed presence of HIV 1 antibodies in serum. 
Treatment for SLE was stopped right away as dealing with co-existing HIV is a complicated task and involves expertise. Therefore, patient was referred to the Infectious Diseases Department of our hospital for further management.

\section{Discussion}

The symptoms of SLE may be constitutional, including fatigue, lethargy, fever and weight loss as those of other autoimmune diseases. It may however involve other systems of the body as well since it is a multi-organ disease. Skin involvement is very common in SLE which includes appearance of malar/discoid rash along with photosensitivity as seen in this patient. According to the SLICC criteria 2012, a person is said to have SLE if he/she meets at least 4 of the 17 with at least one clinical and one immunological criteria [3,4]. We could not carry out coomb's test and complement levels due to lack of availability. Still however, our patient fulfilled the criteria with 8 points.

The concomitant presence of SLE and HIV is rare but common in females as shown by Mody GM and others in their case series. In their study of 13 patients, they showed that HIV can be acquired after the diagnosis of SLE. However, patients with HIV may later develop SLE after the commencement of their anti-retroviral therapy [5]. HIV infection being acquired in a patient after diagnosis of SLE can prove to be fatal. D J Clutterbuck discusses such a case in which the patient after being diagnosed with SLE developed HIV infection, just like our patient did, with a low CD4 cell count. Mycobacterium Avium intracellulare was found in CSF of the patient. Unfortunately the patient died a couple of months later [6].

The same author has reported another case of a patient with clinical diagnosis of SLE who presented with multi drug resistant Tuberculosis and Pneumocystis Carinii and tested positive for HIV antibody [6].

Common etiologies of diarrhea in patients infected with HIV-1 infection include Shigellosis, campylobacter infection and cryptosporidiosis. Some organisms produce diarrhea almost exclusively in HIV1 infected individuals including Mycobacterium Avium Complex and Cytomegalovirus [7].

Treating such patients can become a real challenge as HIV is an immune suppressive condition leading to opportunistic infections. Treatment of HIV patients with anti-retroviral therapy may cause a rapid flare in SLE which may be characterized by cutaneous or other systemic complications [2].

\section{Conclusion}

Co-existence of SLE and HIV in a patient is a life threatening and a dangerous combination that bears a poor prognosis. Such patients require close monitoring.

\section{Consent}

Written informed consent was obtained from the patient's legal guardian for the publication of this case report. A copy of written consent is available for review by the Editor-in-chief of this journal.

\section{Abbreviations}

SLE: Systemic Lupus Erythematosus; HIV: Human Immune Deficiency Virus; CSF: Cerebro Spinal Fluid; CBC: Complete Blood Count; GCS: Glasgow Coma Scale; ESR: Erythrocyte Sedimentation rate; SLICC: Systemic Lupus International Collaborating Clinics.

\section{Competing Interests}

The authors declare that they have no competing interests.

\section{References}

1. Mok CC, Lau CS (2003) Pathogenesis of systemic lupus erythematosus. J Clin Pathol 56: 481-490.

2. Carugati M, Franzetti M, Torre A, Giorgi R, Genderini A, et al. (2013) Systemic lupus erythematosus and HIV infection: A whimsical relationship. Reports of two cases and review of literature. Clin Rheumatol 32: 1399-1405.

3. Manson J, Rahman A (2006) Systemic lupus erythematosus. Orphanet Journal of Rare Diseases 1: 6.

4. Aberle T, Bourn RL, Chen H, Roberts VC, Guthridge JM, et al. (2007) Use of SLICC criteria in a large diverse lupus registry enables SLE classification of a subset of ACR designated subjects with incomplete lupus. Lupus science and medicine 4: 176.

5. Mody GM, Patel N, Budhoo A, Dubula T (2014) Concomitant systemic lupus erythematosus and HIV: case series and literature review. Semin Arthritis Rheum 44: 186-194.

6. Clutterbuck DJ, Watson J, Ruiter AD, Godfrey T, Bradbeer C (2000) Presence of HIV infections in patients diagnosed with systemic lupus erythematosus. Rheumatology 39: 1047-1048.

7. Carcamo C, Hooton T, Noel MH, Weiss NS, Gilman R, et al. (2005) Etiologies and manifestations of persistent diarrhea in adults with HIV-1 infection: a case control study in Lima, Peru. J Infect Dis 191: 11-29. 